\title{
Comparaison de la classification de recettes par trois systèmes de profilage nutritionnel : SAIN,LIM, SENS et 5-C
}

\section{Comparison of recipes classification between 3 French nutrient profiling systems: SAIN,LIM, SENS and 5-C}

\author{
Marion Tharrey $^{\mathrm{a}}$, Anita Houeto ${ }^{\mathrm{a}}$, Christophe Dubois ${ }^{\mathrm{a}, \mathrm{c}}$, Florent Vieux ${ }^{\mathrm{b}}$, Matthieu \\ Maillot $^{\mathbf{b}}$, Nicole Darmon ${ }^{\mathrm{a}, \mathrm{c}}$ \\ ${ }^{a}$ Inserm, Inra 1260, unité mixte de recherche - Nutrition, Obesity and Risk of Thrombosis (UMR \\ NORT), Aix-Marseille université, 13385 Marseille cedex 05, France \\ ${ }^{b}$ MS-nutrition, 13385 Marseille cedex 05, France \\ ${ }^{c}$ INRA 1110, CIRAD, SupAgro, CIHEAM-IAMM, MOISA (Markets, Organizations, \\ institutions and Strategies of Actors), 34060 Montpellier cedex 2, France
}

Correspondant : Nicole Darmon, UMR Moisa, Campus Inra-SupAgro de la Gaillarde ; 2 , place Pierre Viala - Bât. 26 ; 34060 Montpellier Cedex 2, France.

Telephone: +33 633189586. Adresse email: nicole.darmon@inra.fr

\section{RESUME EN FRANÇAIS}

En réponse à l'inscription, dans la loi de santé française, d'un principe d'étiquetage nutritionnel simplifié des aliments, deux systèmes de profilage nutritionnel permettant de classer les aliments sur la base de leur composition nutritionnelle ont été proposés : le système du logo à 5 couleurs $(5-C)$, récemment adopté par le ministère de la Santé et le Système d'Etiquetage Nutritionnel Simplifié (SENS), adapté du système SAIN,LIM, précédemment développé par l'ANSES.

L'objectif de cette étude était de comparer les classements obtenus avec les trois systèmes sur une base de données de recettes constituée pour moitié de plats issus de «La Fabrique à Menus» du PNNS $(n=51)$ et pour moitié de plats servis dans des écoles primaires $(n=50)$. Les coefficients $\mathrm{K}$ de concordance entre les trois systèmes pris deux à deux variaient de 0,44 à 0,57 , avec seulement $35 \%$ de recettes similairement classées par les trois systèmes.

Des discordances de classement sont ainsi mises en évidence entre les trois systèmes de profilage nutritionnel français (SAIN,LIM, SENS et 5-C), bien qu'ils aient tous été développés pour classer les aliments selon leur composition nutritionnelle.

Mot clés : qualité nutritionnelle, aliments, enfants, repas, restauration collective, étiquetage, profils nutritionnels, PNNS 


\section{ABSTRACT}

In response to the inclusion in the French Act on the modernization of the health system of a principle of a simplified nutrition labelling system, two nutrient profiling systems have been developed to classify foods according to their nutritional composition: the 5-colour labelling system (5-C), recently adopted by the French Ministry of Health and the French Simplified Nutritional Labelling System (SENS), adapted from the SAIN,LIM, developed by the ANSES.

The aim of this study was to compare rankings yielded under these three systems based on recipe data $(n=101)$, half of them from an online meal planner developed by The French National Nutrition and Health Program $(n=51)$ and half from meals served in elementary schools $(n=50)$. Side-by-side comparison of the three system using the kappa statistic of agreement showed coefficients varying from 0.44 to 0.57 , with only $35 \%$ of the recipes identically classified by the three systems.

Discrepancies were identified between the three French nutrient profiling systems (SAIN,LIM, SENS and 5-C), although all of them have been developed for the purpose of classifying foods according to their nutritional composition.

Keywords: nutritional quality, foods, children, school catering, labelling, 


\section{INTRODUCTION}

L'augmentation de la prévalence des maladies liées à l'alimentation est un problème de santé publique dans toutes les régions du monde. Dans ce contexte, le profilage nutritionnel - défini par l'OMS comme « la science permettant de classer les aliments en fonction de leur composition nutritionnelle et en vue de promouvoir la santé et prévenir les maladies » (1) - est un outil de choix dans la mise en œuvre de politiques de santé publique visant à influencer positivement les choix alimentaires. Comme évoqué par Lobstein $T$ et al. (2), les champs d'applications des systèmes de profilage nutritionnel sont multiples : accès aux allégations nutritionnelles et de santé ; contrôle de la publicité alimentaire vers les enfants, diffusion de messages clairs et cohérents aux consommateurs; affichage de logos nutritionnels en face avant des emballages ; mise en place de politiques de taxation et/ou subvention des aliments ; incitation de l'industrie agroalimentaire à reformuler les produits afin d'améliorer leur profil nutritionnel.

Dans les pays industrialisés, l'une des utilisations les plus courantes du profilage nutritionnel est l'étiquetage des denrées alimentaires en vue d'aider les consommateurs à adopter des comportements alimentaires plus sains (1). Le règlement (UE) $n^{\circ} 1169 / 2011 \mathrm{du} 25$ octobre 2011 concernant l'information des consommateurs sur les denrées alimentaires (dit INCO) rend obligatoire une déclaration nutritionnelle standardisée et permet sous certaines conditions son accompagnement par un étiquetage simplifié de type graphique ou logo (3). Or afficher un logo nutritionnel sur les produits alimentaires, nécessite en amont d'adopter un système de profilage nutritionnel pour définir les règles de classement des aliments. En France, la loi de Santé prévoit de se conformer au règlement INCO, en adoptant le principe d'un étiquetage nutritionnel simplifié unique sur tout le territoire, qui sera utilisé à titre volontaire par les opérateurs, et dont les modalités précises devront être définies par un décret d'application suivi d'un arrêté (4). Le Système d'Etiquetage Nutritionnel Simplifié (le SENS) et le système du logo à 5 couleurs (le 5-C) ont ainsi été développés et proposés. Ces deux systèmes ont pour point commun de noter les aliments dans leur globalité, mais ils ont des fondements différents : le 5-C, proposé par Hercberg et al. en 2013 par le professeur Serge Hercberg (5), est dérivé du système de profilage anglais FSA-Ofcom (6), tandis que le SENS, proposé en 2015 par un consortium de distributeurs et d'industriels (7), s'appuie sur le système de profilage nutritionnel français $\operatorname{SAIN,LIM}(8,9)$ considéré comme « the French model » par l’OMS en 2008 (1). 
Les choix et les comportements alimentaires, sont influencés par plusieurs facteurs tant au niveau individuel que collectif. En particulier, dans le cadre du Programme National Nutrition Santé, Santé Publique France propose sur son site manger-bouger (10) une interface nommée «La Fabrique à Menus » - dont le but est d'aider les consommateurs à adopter une alimentation plus saine en leur proposant des menus de saison variés et équilibrés. La restauration scolaire est un autre vecteur important d'éducation nutritionnelle. Elle fait l'objet d'un décret et d'un arrêté $(11,12)$, dont les grandes lignes sont dérivées des recommandations élaborées par le Groupe d'étude des marchés de restauration collective et nutrition (GEMRCN) (13). Ainsi, la qualité nutritionnelle de l'offre alimentaire proposée sur le site mangerbouger d'une part, et dans les écoles d'autre part, est en quelque sorte garantie par les repères de consommation alimentaires du PNNS et par les critères du GEM-RCN, respectivement.

L'objectif de la présente étude était d'étudier les concordances et discordances de classement entre le SAIN,LIM, le SENS et le 5-C, sur une base de données de plats principaux respectivement proposés sur l'interface «La Fabrique à Menus » et servis en restauration scolaire. 


\section{DONNEES ET METHODES}

\section{Définitions d'un plat principal et d'une recette}

Ne sont considérées dans cette étude que des recettes composées d'un plat protidique et d'un accompagnement, combinaison désignée par la suite sous le terme de «plat principal». Chaque recette était caractérisée par son nom, la liste des ingrédients, la méthode de préparation et de dressage des plats, et la quantité servie par personne. Lors de la sélection des recettes, les dates ont été choisies de manière à n'avoir que des recettes proposées pour les périodes d'hiver ou de printemps, tout en évitant les jours de fêtes. Les sandwichs, les soupes, les plats préparés industriels, et les doublons ont été exclus de l'étude. Pour la restauration scolaire l'échantillon de recettes fourni correspondait à la période automne/hiver.

\section{Création de la base de données de recettes}

\section{Sélection des recettes de l’interface «La Fabrique à Menus »}

La sélection des recettes de «La Fabrique à Menus », a été réalisée à partir du site de mangerbouger (10). Des menus hebdomadaires ont été obtenus en sélectionnant parmi les critères de recherche proposés sur l'interface : 7 jours ; 4 personnes ; déjeuner et dîner. L'opération a été répétée 4 fois, aboutissant à 56 plats principaux (1 plat principal par repas, 2 repas par jour, 4 semaines de 7 jours). Sur les 56 recettes, 5 recettes étaient répétées, amenant à un total de 51 recettes différentes les unes des autres.

\section{Sélection des recettes de la restauration scolaire}

Dans le cadre d'une étude coordonnée par le Centre d'information sur les viandes (CIV) en 2015/2016, une table de recettes avait été transmise par des adhérents de Resto'Co et des sociétés adhérentes au SNRC (Syndicat national de la Restauration Collective), aux investigateurs de cette étude. L'ensemble des recettes provenaient de 20 établissements primaires des régions Centre, Midi-Pyrénées, Rhône-Alpes, Pays de la Loire, Île-de-France, PACA, Grand Est, Bretagne, Sud-Ouest, Sud Est, Aquitaine, Lorraine et Nord-Pas-de-Calais. L'âge des élèves s'étendait de 4 à 13 ans, 97\% d'entre eux ayant entre 6 et 10 ans (14). La table comportait un total de 3955 plats dont 1968 sur la période automne-hiver. Parmi les 1968 on décomptait 400 accompagnements et 400 plats protidiques. C'est sur ce dernier échantillon de 800 recettes qu'une demande a été faite afin d'obtenir une liste de 50 plats principaux sélectionnés aléatoirement (25 de Resto'Co et 25 du SNRC). 


\section{Calcul de la composition nutritionnelle des recettes}

Afin d'obtenir la composition nutritionnelle de chaque recette, nous avons utilisé le logiciel Loginuts (15) porté par le Ministère de l'Agriculture, de l'Agroalimentaire et de la Forêt. Les informations suivantes ont été saisies pour chaque recette : nom de la recette, ingrédients, poids de chaque ingrédient en grammes et nombre de convives. Pour le sel ou l'huile, les quantités n'étaient pas toujours précisément indiquées. Dans ce cas, une quantité standard, utilisée dans le domaine de la restauration collective, a alors été appliquée. Pour le sel: $5 \mathrm{~g} / \mathrm{kg}$ (bœuf, agneau, veau); $4 \mathrm{~g} / \mathrm{kg}$ (porc); $6 \mathrm{~g} / \mathrm{kg}$ (volaille, abats); $3 \mathrm{~g} / \mathrm{kg}$ (poisson): $3 \mathrm{~g} / \mathrm{kg}$ (légumes); $6 \mathrm{~g} / \mathrm{kg}$ (riz, pâtes, semoule); $5 \mathrm{~g} / \mathrm{kg}$ (sauces); pour l'huile: $30 \mathrm{ml} / \mathrm{kg}$ (viande), $60 \mathrm{ml} / \mathrm{kg}$ (accompagnements) et $10 \mathrm{ml} / \mathrm{kg}$ (poisson). Pour les ingrédients quantifiés en nombre (ex : 1 carotte, 1 cuillérées de poivre, 1 noix de beurre...), un poids usuel a été utilisé (par ex., 1 carotte $=100 \mathrm{~g}$ ). Les grammages exprimés sous la forme d'un intervalle ont également été convertis en poids moyen (par exemple, dans le cas d'un intervalle 100-150g, le poids saisi était de $125 \mathrm{~g})$.

Les recettes indiquent le poids des aliments tel que achetés, alors que les convives consomment les aliments préparés. Un coefficient de conversion "acheté=>consommé" - a donc été appliqué pour tenir compte des modifications de poids lors de la préparation des aliments (épluchage, parage, cuisson, réhydratation...). Enfin, la composition nutritionnelle du plat tel que consommé (pour 100g de plat) a été estimée à partir de la table de composition alimentaire Ciqual 2013, intégrée au logiciel Loginuts (16).

\section{Présentation des trois systèmes de profilage nutritionnel}

\section{Système SAIN,LIM}

Le système SAIN,LIM, a été développé en France, en 2008, dans le cadre d'un groupe de travail de l'ANSES visant à contrôler l'accès aux allégations nutritionnelles et de santé. Il a été construit à partir de scores préexistants : le SAIN (ou score d'adéquation individuel aux recommandations nutritionnelles) et le LIM (ou score des nutriments à limiter) $(8,9)$. Le SAIN estime le pourcentage moyen d'adéquation aux apports nutritionnels conseillés en 5 nutriments favorables (protéines, fibres, calcium, fer, vitamine C), dans 100kcal d'aliment ; c'est une densité nutritionnelle (richesse en nutriments essentiels pour 100kcal). La vitamine $\mathrm{D}$ est prise en compte quand elle est présente de façon significative. Le LIM estime les aspects défavorables de l'aliment à travers le pourcentage moyen d'excès par rapport aux valeurs maximales recommandées en sodium, sucres ajoutés et acides gras saturés, dans $100 \mathrm{~g}$ 
d'aliment. Un seuil sur chaque score permet de définir 4 classes qui peuvent être visualisées par représentation graphique des composantes positives et négatives (score LIM en abscisse et score SAIN en ordonnée). Le système SAIN,LIM est transversal, c'est-à-dire que les mêmes calculs s'appliquent à tous les aliments. Deux exceptions sont néanmoins considérées en fonction de leurs densités énergétiques extrêmes: les boissons (leur LIM est multiplié par 2,5) et les matières grasses et fruits oléagineux (des nutriments lipidiques sont pris en compte dans le SAIN).

\section{Système SENS}

Le système SAIN,LIM a été adapté pour l'étiquetage et modifié pour servir d'algorithme au Système d'Etiquetage Nutritionnel Simplifié (SENS) qui, comme le système SAIN,LIM, répartit les aliments en quatre classes sur la base des scores $\operatorname{SAIN}_{\mathrm{SENS}}$ et $\operatorname{LIM}_{\mathrm{SENS}}(7,17)$. Le $\mathrm{SAIN}_{\mathrm{SENS}}$, composante «positive», intègre des paramètres nutritionnels considérés comme qualifiants (protéines, fibres, teneur en fruits et légumes). Des pondérations et/ou des paramètres additionnels (calcium ou vitamine $\mathrm{C}$ ou nutriments lipidiques) sont pris en compte en fonction de la catégorie ou sous-catégorie considérée permettant ainsi de tenir compte des spécificités nutritionnelles des différentes catégories (ex : valorisation des fibres pour les produits céréaliers). Les fruits et légumes tels que définis par le système SENS n'incluent pas les tubercules, les fruits oléagineux, les fruits secs ni les légumes secs. Le LIMSENS, composante «négative», intègre des paramètres nutritionnels considérés comme disqualifiants, à savoir sodium, acides gras saturés et sucres libres.

Des seuils principaux (les mêmes que ceux du système SAIN,LIM) et des scores secondaires appliqués sur les scores SAIN $_{\mathrm{SENS}}$ et LIM $_{\mathrm{SENS}}$ définissent 4 classes ordonnées. Comme pour le système SAIN,LIM, le positionnement d'un aliment sur un graphe bidimensionnel $\left(\mathrm{LIM}_{\mathrm{SENS}}\right.$ en abscisse et $\mathrm{SAIN}_{\text {SENS }}$ en ordonnée) permet de visualiser ses composantes positives et négatives, ainsi que la classe à laquelle il appartient.

\section{Système 5 Couleurs}

Adapté du score FSA-Ofcom $(6)$, le système 5-C $(18,19)$ est basé sur un score négatif, le $a$ score, et un score positif, le c-score. Le a-score tient compte de quatre paramètres : la densité énergétique $(\mathrm{kcal} / 100 \mathrm{~g})$, les acides gras saturés (ou le rapport acides gras saturés sur lipides totaux), le sodium et les sucres totaux, chaque paramètre étant noté de 0 à 10 . Les points 
obtenus pour chacun des quatre paramètres sont ensuite additionnés pour obtenir le a-score. Le $c$-score est calculé sur la base de trois paramètres : la teneur en fruits et légumes, en fibres et en protéines, chaque paramètre étant noté sur une échelle de 0 à 5 . La définition de fruits et légumes selon le système 5-C, à la différence du système SENS, inclut les fruits à coque, les fruits secs ou séchés et les légumes secs, pourvu qu'ils soient considérés comme non altérés par un éventuel procédé de transformation. Les points obtenus pour chacun des trois paramètres sont ensuite additionnés pour obtenir le c-score. La détermination des composantes positives, négatives et du score final est dépendante de la catégorie à laquelle appartient l'aliment (matières grasses, fromages, boissons, autres).

Le score final de l'algorithme 5-C est déterminé par soustraction de la composante positive à la composante négative avec certaines spécificités de calcul selon la catégorie d'aliment considérée. Des seuils appliqués sur le score final définissent 5 classes auxquelles sont attribuées 5 couleurs différentes; du vert (classe A, la meilleure) au rouge (classe D, la moins bonne).

\section{Calcul du profil nutritionnel des recettes}

Chaque recette a été rattachée à une des catégories définie par le SENS et le 5-C. Les scores des systèmes SAIN,LIM (SAIN et LIM), SENS (SAIN SENS $_{\text {et }}$ LIM $_{\text {SENS }}$ ), et 5-C (c-score et ascore) ont ensuite été calculés et la classe correspondante identifiée. Un numéro a été attribué à chaque classe (de 1 à 4 pour le SAIN,LIM et le SENS et de 1 à 5 pour le 5-C); les aliments de bonne qualité nutritionnelle étant en classe 1 et ceux à limiter en classe 4 (respectivement 5 pour le 5-C). Une projection des recettes avec les couleurs du 5-C sur le plan SAIN,LIM et sur le plan SENS a permis de visualiser les différences de classement entre les 3 systèmes.

\section{Analyses statistiques}

La concordance entre les trois systèmes a été statistiquement évaluée avec le test de Kappa pondéré, qui permet de tenir compte de la «gravité» des discordances. Par exemple, une discordance d'une seule classe sera considérée comme moins « grave » qu'une discordance de deux ou trois classes. La différence entre les variables quantitatives a été évaluée à l'aide du test de Wilcoxon - Mann Whitney. Les analyses statistiques ont été réalisées à l'aide des logiciels SAS 9.4 et IBM SPSS Statistics 20. Un seuil de significativité à 5\% a été retenu pour l'ensemble des tests. 


\section{RESULTATS}

Aucune recette de notre échantillon n'appartenait à la dernière classe (classe 5) du système 5C. Toutes les recettes étaient donc réparties en 4 classes par les trois systèmes. La Figure 1 représente la répartition des 101 recettes dans les 4 classes des trois systèmes de profilage nutritionnel SAIN,LIM, SENS et 5-C. Le SAIN,LIM a affiché le taux le plus élevé de recettes en classe $4(\mathrm{~N}=16 ; 15,8 \%)$ et le système 5 -C le taux le plus élevé de recettes en classe 1 $(\mathrm{N}=56 ; 55,4 \%)$. Les classes intermédiaires étaient d'avantage représentées par le système SENS (classes 2 et 3 avec respectivement 33,7\% et 13,9\% des recettes).

Les tests de kappa (évaluant la concordance entre les systèmes sur l'ensemble des recettes) ont mis en évidence des coefficients variant de 0,44 à 0,57 entre les trois systèmes pris deux à deux. La concordance la plus élevée a été observée entre le SENS et le 5-C ( $\mathrm{K}=0,57$; $\mathrm{p}<0,001)$, suivie par la concordance entre le SAIN,LIM et le 5-C $(\mathrm{K}=0,45 ; \mathrm{p}<0,001)$, et enfin entre le SAIN,LIM et le SENS $(\mathrm{k}=0,44 ; \mathrm{p}<0,001)$. Seulement 35 recettes sur les $101(34,7 \%)$ ont été classées de façon strictement similaire par les trois systèmes, avec $28(27,7 \%), 6$ $(5,9 \%), 1(1,0 \%)$ et 0 recettes dans les classes $1,2,3$ et 4 respectivement, indépendamment du système de profilage appliqué.

Une projection des 101 recettes sur les plans SAIN,LIM et SENS, selon le code couleur du système 5-C est présentée Figure 2. Les recettes numérotées sont celles pour lesquelles des discordances de plus d'une classe ont été observées entre les deux systèmes comparés. En projetant sur le plan du SAIN,LIM (Figure 2A), les écarts observés étaient majoritairement de 1 classe (45 recettes, soit 44,6\%), suivi par des écarts de 2 classes pour 4 recettes (4,0\%), et de 3 classes pour 1 recette $(1,0 \%)$. La projection sur le plan du SENS a atténué ces discordances (Figure 2B) ; 35 recettes $(34,7 \%)$ ont affiché un écart de 1 classe, aucune recette n'ayant un écart supérieur à 1 classe. Enfin, en comparant le SAIN,LIM et le SENS, les divergences étaient de 1 classe pour 43 recettes $(42,6 \%)$ et de 2 classes pour 6 recettes $(5,9 \%)$.

Les caractéristiques nutritionnelles des deux séries de recettes sont présentées dans la Figure 3. Etant destinées à des enfants, les recettes de la restauration scolaire étaient présentées en plus faibles portions et apportaient moins de calories que les recettes de «La Fabrique à Menus » mais la densité énergétique moyenne était similaire entre les deux séries de recettes $(\mathrm{p}=0,615)$. Les recettes de «La Fabrique à Menus» avaient une plus faible teneur en nutriments à limiter (plus faible LIM, $\mathrm{p}=0,002$ ) que celles de la restauration scolaire. 


\section{DISCUSSION}

Cette étude a comparé trois systèmes de profilage nutritionnel développés en France dans le but de classer les aliments en fonction de leur composition nutritionnelle. Les résultats ont mis en évidence des divergences de classement notables entre les différents systèmes de profilage. Seulement $34,7 \%$ des recettes étaient similairement classées par les trois systèmes. La concordance portait surtout sur les aliments de meilleur profil nutritionnel, avec 28 recettes sur les 35 identiquement classées qui appartenaient à la classe 1 de chacun des systèmes. La concordance la plus forte a été observée entre le SENS et le 5-C, tandis que le système SAIN,LIM s'est avéré classer plus sévèrement que les deux autres systèmes.

La construction des scores, le choix des nutriments, la prise en compte de catégories, les références nutritionnelles et les seuils utilisés sont autant d'éléments pouvant expliquer les variations observées entre le SAIN,LIM, le SENS et le 5-C. L'attribution d'une classe, par le système 5-C, dépend d'un score final obtenu par compensation de la composante défavorable par la composante favorable. En revanche, les systèmes SAIN,LIM et SENS sont bidimensionnels: les scores positifs et négatifs restent indépendants l'un de l'autre. Une analyse des scores sous-jacents a montré que le score final du 5-C était positivement et fortement corrélé aux scores négatifs : LIM, LIM ${ }_{\text {SENS }}$ et a-score $\left(r_{s}=0,78 ; r_{s}=0,79\right.$ et $r_{s}=0,82$ respectivement ; $<<0,001)$, et plus faiblement corrélé aux scores positifs : SAIN, SAIN SENS $_{\text {et }}$ c-score $\left(r_{s}=-0,42 ; r_{s}=-0,49\right.$ et $r_{s}=-0,37$ respectivement ; $\left.p<0,001\right)$. Des résultats similaires ont été obtenus dans le rapport de l'ANSES comparant les systèmes SENS et 5-C (20) et dans l'étude de Drewnowski et al. (21) évaluant la relation entre les systèmes SAIN,LIM et FSAOfcom (système sur lequel s'est fondé le 5-C). Par conséquent, le classement 5-C serait donc d'avantage expliqué par la composante négative des recettes.

Une meilleure concordance a été observée entre le 5-C et le SENS qu'entre le SENS et le SAIN,LIM, bien que les sous scores composants ces deux derniers systèmes (SAIN,

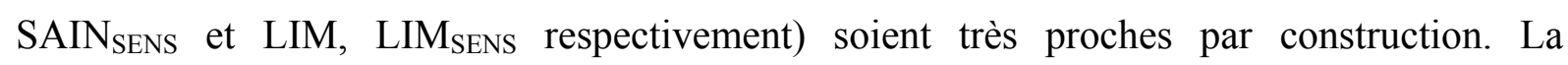
représentation graphique des aliments sur les plans du SAIN,LIM et du SENS a permis de mettre en évidence l'importance du choix des seuils dans la détermination des classes. L'attribution de seuils secondaires dans le SENS a conduit à un classement ordonné et progressif coïncidant d'avantage avec les classes du 5-C, tout en limitant le saut de classe par simple effet de bord comme cela était le cas pour le SAIN,LIM. 
Les 3 systèmes de profilage nutritionnel présentent plusieurs avantages respectifs. Les forces des systèmes SAIN,LIM et SENS sont principalement l'intégration dans leur construction des recommandations nutritionnelles officielles, la prise en compte de la densité nutritionnelle des aliments (représentée par le SAIN) et l'utilisation de scores qui ont une dimension compréhensible par tous (\% d'adéquation pour le SAIN et \% d'excès pour le LIM). La majorité des données relatives au calcul du 5-C fait partie de la déclaration nutritionnelle obligatoire dans le cadre du règlement INCO (3), facilitant ainsi la collecte des informations, à l'exception de la teneur en fruits et légumes et en fibres, qui ne font pas l'objet d'une déclaration obligatoire. Ainsi, quel que soit le système considéré, son applicabilité reste conditionnée par la disponibilité des données.

La présente étude visait également à comparer les profils nutritionnels de séries de recettes provenant de supports vecteurs d'éducation nutritionnelle; à savoir l'interface « La Fabrique à Menus » du PNNS, et la restauration scolaire. Développée dans le cadre du PNNS, «La Fabrique à Menus », propose des recettes de bon profil nutritionnel, la quasi-totalité de l'échantillon étant classée favorablement (classe 1 ou 2). Ces résultats étaient attendus puisque «La Fabrique à Menus » vise à proposer « des menus de saison variés pour manger équilibré toute la semaine en accord avec les repères nutritionnels du Programme National Nutrition Santé ». Les recettes du PNNS avaient d'ailleurs un LIM significativement plus faible que celles de la restauration scolaire. Il est toutefois intéressant de noter que ces recettes présentaient aussi un SAIN relativement faible, de 5,2\%/100kcal en moyenne, soit à peine supérieur au seuil de 5\% (correspondant à 100\% d'adéquation pour $2000 \mathrm{kcal}$ ). Il semblerait donc que le PNNS promeut des régimes alimentaires équilibrés dans lequel l'accent est mis en priorité sur la limitation de la consommation de nutriments défavorables avec une plus faible considération de la densité nutritionnelle des plats proposés.

Dans un souci de prévention de l'obésité infantile, la restauration scolaire fait l'objet d'un décret visant à garantir la qualité nutritionnelle de l'offre alimentaire qui y est proposée (12). Nos résultats suggèrent l'impact positif de ces règles nutritionnelles. En effet, dans notre échantillon de plats principaux servis en restauration scolaire, les recettes, dans leur grande majorité, étaient classées favorablement (classe 1 ou 2). En accord avec le fait que l'équilibre nutritionnel ne se réalise ni sur un repas, ni même sur une journée, le décret prévoit des fréquences de service minimales et/ou maximales de certains plats sur la base de 20 repas successifs. Ainsi, le service de recettes classées défavorablement (classe 3 et 4 ) ne met pas en 
péril l'équilibre global car elles sont contrebalancées par les recettes de meilleure qualité nutritionnelle.

Les déterminants des choix alimentaires sont complexes et multiples (22). En particulier la publicité alimentaire aurait un impact important, et la promotion d'aliments de mauvaise qualité nutritionnelle engendrerait des comportements alimentaires néfastes $(23,24)$. Face à la diversité des informations médiatiques ayant trait à l'alimentation et considérant leur influence sur les choix alimentaires, il est attendu d'un système graphique d'étiquetage nutritionnel simplifié tel que prévu par l'article 35 du règlement INCO qu'il aide les consommateurs à comparer les produits entre eux de façon à identifier les plus favorables à la santéf(3). Pourtant l'ANSES a récemment estimé comme incertaine la capacité d'une telle mesure à améliorer effectivement les choix des consommateurs (25). En fait, c'est en conditions réelles d'achat que l'impact des logos nutritionnels est le plus difficile à démontrer (26). Une étude réalisée pendant 6 mois dans des magasins situés dans des quartiers défavorisés à Marseille a pourtant mis en évidence l'intérêt d'un logo signalant les aliments de bonne qualité nutritionnelle (estimée par le système SAIN,LIM) et peu coûteux (27). Plus récemment, une expérimentation de 10 semaines dans 60 magasins en France a comparé l'impact sur la qualité nutritionnelle (estimée par le score FSA-Ofcom) de 4 logos nutritionnels (28). Les résultats, communiqués par le Conseil Scientifique du projet, ont conclu à l'efficacité de 3 systèmes sur 4, parmi lesquels figuraient les logos Nutri-Score et SENS, respectivement fondés sur les systèmes de profilage nutritionnel 5-C et SENS. Le Nutri-Score a engendré une amélioration de 4,4\% et le SENS de 3,3\%. Cette faible différence entre les deux logos était statistiquement significative et pourrait s'expliquer par une meilleure compréhension par les consommateurs du design et des couleurs de Nutri-Score que ceux du SENS (96\% des consommateurs ont correctement ordonné les étiquettes du logo Nutri-Score vs 79\% pour le logo SENS), mais aussi par le fait que le score même sur lequel est fondé le Nutri-Score (le FSA-Ofcom) a été utilisé pour démontrer la supériorité de ce logo sur les autres, et enfin par le fait qu'une égalité de traitement entre les 4 systèmes n'a pas pu être garantie lors de l'étude, compte tenu de la très large médiatisation positive dont a fait l'objet le Nutri-Score sur l'ensemble du territoire. Les résultats d'une autre étude comparative, réalisée par Crosetto et al., et utilisant les méthodes de l'économie expérimentale en laboratoire (29) ont également donné Nutri-Score en tête face à quatre autres systèmes d'étiquetage nutritionnels dont le SENS, mais en utilisant à nouveau le score FSAOfcom comme critère principal de jugement. Sur la base des résultats de l'expérimentation en 
condition réelles (28) et de l'économie expérimentale (29), Marisol Touraine, Ministre des Affaires sociales et de la Santé, a considéré que l'intérêt des logos pour améliorer la qualité nutritionnelle du panier d'achat des consommateurs était démontré, ainsi que l'efficacité du logo Nutri-score comparativement aux autres systèmes d'étiquetage nutritionnel (30).

Dans son rapport sur le profilage nutritionnel, l'OMS reconnait qu'une limite importante du concept de profilage nutritionnel est qu'il n'existe pas de «gold-standard» permettant d'apprécier objectivement la qualité nutritionnelle d'un produit alimentaire considéré isolément (1). Positionner les aliments les uns par rapport aux autres en fonction de leur qualité nutritionnelle reste donc une science subjective, et la pertinence des différentes méthodologies proposées fait encore l'objet de nombreux débats (25). Or, une des conditions d'application de l'article $35 \mathrm{du}$ règlement $\mathrm{INCO}$ est l'alinea «f» qui stipule que les formes graphiques complémentaires au tableau nutritionnel obligatoire «sont objectives (et non discriminatoires) »(3). Fonder ces formes graphiques complémentaires sur des systèmes de profilage notant les aliments dans leur globalité semble donc incompatible avec le respect de l'article 35 puisque ces systèmes peuvent noter différemment les mêmes aliments (20,31), ainsi que le confirme la présente étude.

\section{CONCLUSION}

Les trois systèmes de profilage nutritionnel français (SAIN,LIM, SENS et 5-C) peinent à classer les recettes de la même manière, bien qu'ils aient tous été développés pour classer les aliments selon leur composition nutritionnelle. En l'absence de «gold-standard», nos résultats ne nous permettent pas de juger de la pertinence d'un système par rapport à un autre. 


\section{Références}

1. WHO. Nutrient Profiling: Report of a WHO/IASO Technical Meeting London, United Kingdom, 4-6 October 2010. Geneva: WHO; 2011.

2. Lobstein T, Davies S. Defining and labelling "healthy" and "unhealthy" food. Public Health Nutr. 2009 Mar;12(3):331-40.

3. The European Parliament and the Council of European Union.Regulation (EU) No 1169/2011 of the European Parliament and of the Council of 25 October 2011 on the provision of food information to consumers, amending Regulations (EC) No 1924/2006 and (EC) No 1925/2006 of the European Parliament and of the Council. Off $J$ Eur Union 2011;304(18-63).

4. Marisol Touraine. Projet de Loi relatif à la Santé. NOR : AFSX1418355L/Bleue-1. ETUDE D'IMPACT. Republique Française, 14 octobre 2014.

5. Hercberg S, Julia C. Propositions pour un nouvel élan de la politique nutritionnelle française de santé publique dans le cadre de la Stratégie Nationale de Santé Introduction générale. 15 novembre 2013.

http://www.ladocumentationfrancaise.fr/var/storage/rapports-publics/144000068.pdf (accédée le 18 avril 2017)

6. Rayner M, Scarborough P, Stockley L, Boxer A. Nutrient profiles: Further refinement and testing of Model SSCg3d. Final report. September 2005.

http://www.food.gov.uk/multimedia/pdfs/npreportsept05.pdf. (accédée le 18 avril 2017)

7. Darmon N, Maillot M, Braesco V, Tafournel E. L'Algorithme du Système d'Etiquetage Nutritionnel Simplifié (SENS). Développement, description et validation. Rapport remis le 23 décembre 2015 à l'Agence nationale de sécurité sanitaire de l'alimentation, de l'environnement et du travail (ANSES).

8. $\quad$ AFSSA. Définition de profils nutritionnels pour l'accès aux allégations nutritionnelles et de santé : propositions et arguments. Paris: AFSSA; 2008.

9. Darmon N, Vieux F, Maillot M, Volatier JL, and Martin A. Nutrient profiles discriminate between foods according to their contribution to nutritionally adequate diets: a validation study using linear programming and the SAIN,LIM system. $A m J$ Clin Nutr 2009;89:1227-36.

10. PNNS. La Fabrique à menus | Manger Bouger. http://www.mangerbouger.fr/MangerMieux/Vos-outils/Fabrique-amenus?gclid=CjwKEAiA7ejCBRDlp8uF6ezPnjoSJAAPED7M7pzXwTCe3ZZFgPM8 fmTA7xdOn3odXF9pmmHGesmddBoC3aPw_wcB\#xtor=SEC-9-GOO-[Marque]--S[la fabrique à menu] (accédée le 18 avril 2017)

11. AFSSA. AVIS de l'Agence française de sécurité sanitaire des aliments relatif à un projet de décret et à un projet d'arrêté concernant la qualité nutritionnelle des repas servis dans le cadre de la restauration scolaire. Saisine $n^{\circ}$ 2008-SA-0394. 2009

12. JORF $n^{\circ} 0229$ du 2 octobre 2011 page 16572 texte $n^{\circ} 29$. Décret $n^{\circ} 2011-1227 d u 30$ septembre 2011 relatif à la qualité nutritionnelle des repas servis dans le cadre de la restauration scolaire. NOR: AGRG1032342D 
https://www.legifrance.gouv.fr/eli/decret/2011/9/30/AGRG1032342D/jo/texte (accédée le 18 avril 2017)

13. Groupe d'Etude des Marchés de Restauration Collective et de Nutrition (GEMRCN). Ministère de l'Economie,des Finances et de l'industrie (Minefi).Direction des Affaires Juridiques Observatoire Economique de l'Achat Public. Recommandation Nutrition Complétée et mise à jour au 10 octobre 2011. Collect Marchés Publics 2011. http://www.economie.gouv.fr/directions_services/daj/marches_publics/oeap/gem/table. htm (accédée le 18 avril 2017)

14. Ministère de l'éducation nationale de l'enseignement supérieur et de la recherche. Repères et références statistiques sur les enseignements, la formation et la recherche. 2014.

http://cache.media.education.gouv.fr/file/2014/04/7/DEPP_RERS_2014_344047.pdf (accédée le 18 avril 2017)

15. Ministère de l'agriculture de l'agroalimentaire et de le forêt. LOGINUTS : un logiciel pour vous aider à respecter les règles nutritionnelles en restauration scolaire.

Améliorer la restauration scolaire. Alim'agri. http://agriculture.gouv.fr/ameliorer-larestauration-scolaire (accédée le 18 avril 2017

16. ANSES. Table Ciqual 2013. https://www.anses.fr/fr/content/table-ciqual-2013 (accédée le 25 janvier 2017)

17. Tharrey M, Maillot M, Braesco V, Darmon N. From the SAIN,LIM system to the SENS algorithm: a review of a French approach to nutrient profiling. Proc Nutr Soc doi:10.1017/S0029665117000817 (in press).

18. Julia C, Péneau S, Ducrot P, Deschamps V, Méjean C, Touvier M, et al. Application aux produits disponibles sur le marché français du profil nutritionnel associé au système 5 couleurs $(5-\mathrm{C})$ : cohérence avec les repères de consommation du PNNS. Cah Nutr Diététique 2015;50(4):189-201.

19. Ducrot P, Julia C, Méjean C, Kesse-Guyot E, Touvier M, Fezeu LK, et al. Impact of Different Front-of-Pack Nutrition Labels on Consumer Purchasing Intentions. Am J Prev Med 2016;50(5):627-36.

20. ANSES. Faisabilité de la classification des aliments selon l'algorithme proposé par la FCD Comparaison des résultats obtenus à ceux du système 5-C intégrant les ajustements du HCSP. Paris: ANSES; 2016.

21. Drewnowski A, Maillot M, Darmon N. Testing nutrient profile models in relation to energy density and energy cost. European Journal of Clinical Nutrition 2009;63, 674683.

22. Booth SL, Sallis JF, Ritenbaugh C, Hill JO, Birch LL, Frank LD, et al. Environmental and Societal Factors Affect Food Choice and Physical Activity: Rationale. Nutr Rev 2001;59(3):S21-39.

23. Zimmerman FJ, Shimoga S V. The effects of food advertising and cognitive load on food choices. BMC Public Health 2014;14:342.

24. Boyland EJ, Nolan S, Kelly B, Tudur-Smith C, Jones A, Halford JC, et al. Advertising as a cue to consume: a systematic review and meta-analysis of the effects of acute exposure to unhealthy food and nonalcoholic beverage advertising on intake in children 
and adults. Am J Clin Nutr 2016;103(2):519-33.

25. ANSES. AVIS relatif à l'analyse de la pertinence en matière de nutrition de systèmes d'information nutritionnelle destinés au consommateur. Paris: ANSES; 2017

26. Gaigi H, Raffin S, Maillot M, Adrover L, Ruffieux B, Darmon N. Expérimentation d'un fléchage nutritionnel dans deux supermarchés à Marseille « Le choix Vita+». Cah Nutr Diététique 2015;50(1):16-24.

27. Gamburzew A, Darcel N, Gazan R, Dubois C, Maillot M, Tomé D,et al. Manger Top, une démarche de marketing social bénéfique en milieu défavorisé. Cah Nutr Diet 2017 Doi : 10.1016/j.cnd.2017.03.006 [accepté le 28 mars 2017, in press].

28. Evaluation Ex Ante de systèmes d'étiquetage nutritionnel graphique simplifié. Rapport final du comité scientifique, 15 mars 2017. http://socialsante.gouv.fr/IMG/pdf/rapport_comite_scientifique_etiquetage_nutritionnel_150317.p df (accédée le 18 avril 2017)

29. Crosetto P, Lacroix A, Muller L, Ruffieux B. Modification des achats alimentaires en réponse à cinq logos nutritionnels. Cah Nutr Diet 2017 doi :10.1016/j.cnd.2017.04.002 [accepté le 19 avril 2017, in press].

30. Ministère des Affaires Sociales et de la Santé. Marisol Touraine se félicite des résultats des études sur l'impact d'un logo nutritionnel : leur intérêt et l'efficacité du logo Nutriscore sont démontrés. Communiqué de Presse, Paris, le 15 mars 2017. Communiqué de Presse, Paris; 2017. http://social-sante.gouv.fr/actualites/presse/communiques-depresse/article/marisol-touraine-se-felicite-des-resultats-des-etudes-sur-l-impact-d-unlogo (accédée le 18 avril 2017)

31. Azaïs-Braesco V, Goffi C, Labouze E. Nutrient profiling: comparison and critical analysis of existing systems. Public Health Nutr 2006 Aug;9(5):613-22. 


\section{REMERCIEMENTS}

Les auteurs remercient le CIV Viande, Sciences et Société et ses partenaires, de leur avoir permis d'utiliser la table de recettes collectées auprès de structures adhérentes au SNRC (Syndicat National de la Restauration Collective) et à Restau'co (Réseau de professionnels de la restauration collective en gestion directe) dans le cadre d'une étude sur la qualité nutritionnelle des repas servis en école primaire.

Déclaration d'intérêt : ND et MM ont développé l'algorithme du SENS dans un précédent projet financé par plusieurs compagnies et secteurs de l'agroalimentaire (Atla, Auchan Retail, Boissons rafraîchissantes de France, Carrefour, Casino, Findus, Fleury Michon, Marie Sablé, Monoprix, Système U, Unijus). Aucun de ces détaillants et entreprises n'a été impliqué dans la présente étude.

Florent Vieux est employé de MS-Nutrition, société qui a reçu un financement dans le cadre des travaux de développement et validation du SENS.

Marion Tharrey, Anita Houeto et Christophe Dubois n'ont pas de lien d'intérêt avec cette étude. 
Figure 1. Répartition de recettes $(n=101)$ dans les 4 classes pour chacun des trois systèmes de profilage nutritionnel : SAIN,LIM, SENS et 5-C.

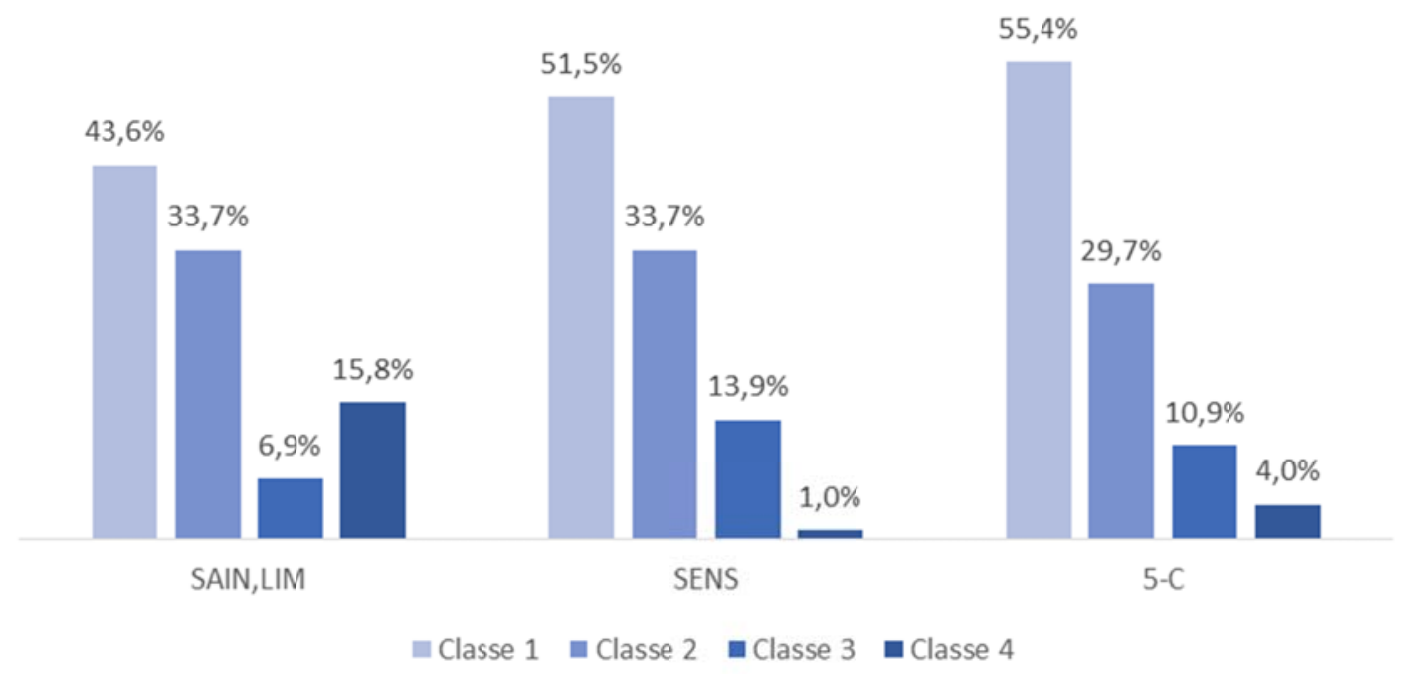


Figure 2. Projection des recettes $(n=101)$ avec les couleurs du $5-C$ sur le plan SAIN,LIM (2A) et sur le plan SENS (2B). Les recettes numérotées sont celles pour lesquelles une discordance de plus d'une classe est observée entre les deux systèmes comparés.

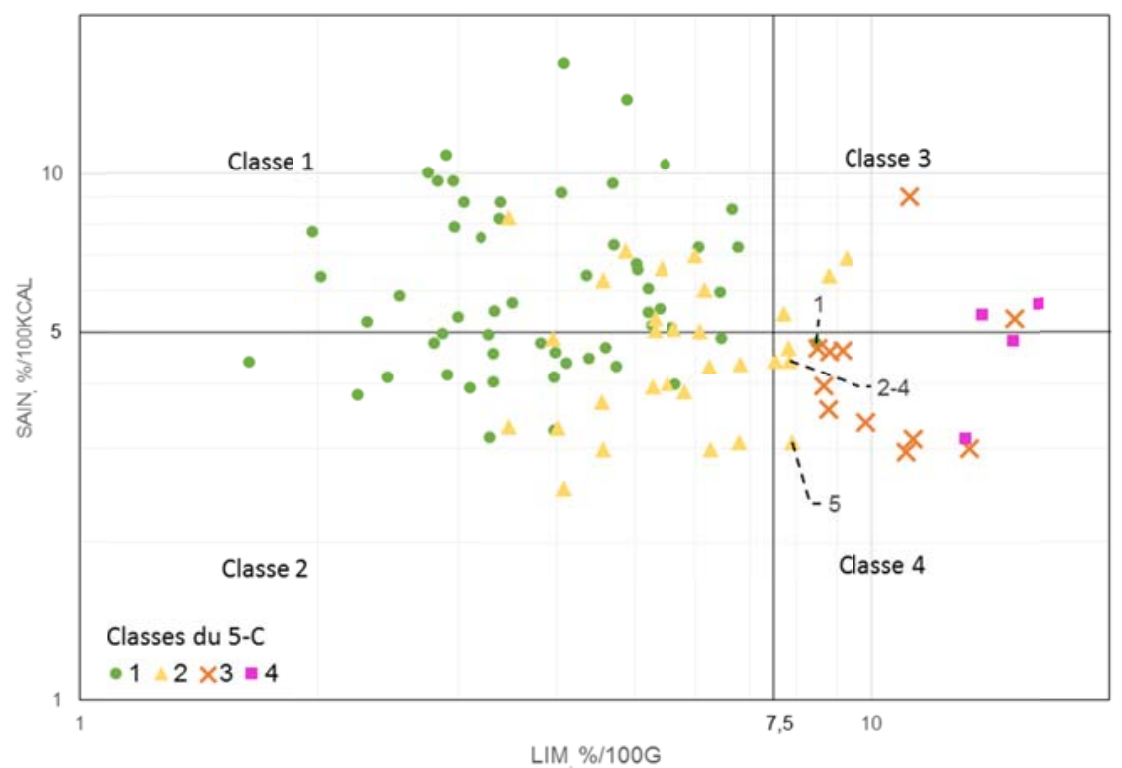

2A) Projection des aliments sur le plan SAIN,LIM avec les couleurs du système 5-C

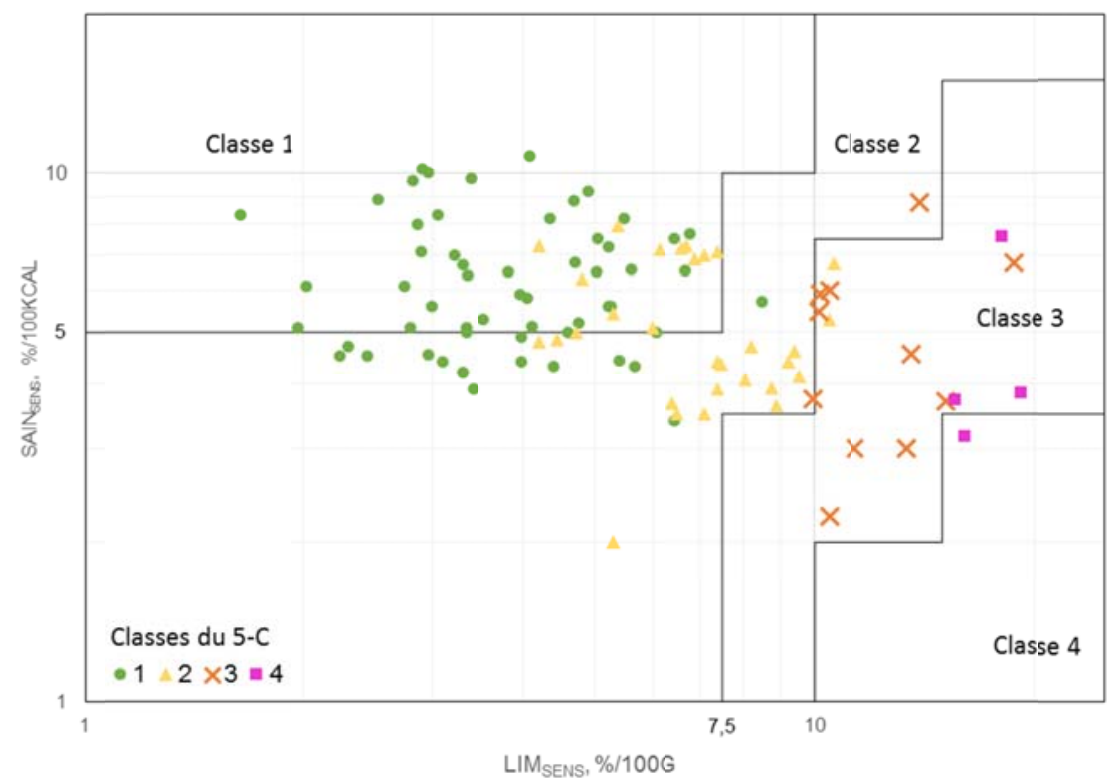

2B) Projection des aliments sur le plan SENS avec les couleurs du système 5-C

Recettes ayant un écart de classement entre les systèmes supérieur à une classe :

1 : Cordon bleu - poêlée méridionale

2 : Flamenkush - salade

3 : Aiguillette de poulet - gratin de pomme de terre

4 : Lasagnes à la bolognaise

5 : Couscous poulet merguez-semoule 
Figure 3. Caractéristiques nutritionnelles et classement des deux séries de recettes - « La Fabrique à Menus » $(n=51)$ et la restauration scolaire $(n=50)$ - par les trois systèmes de profilage nutritionnel : SAIN,LIM, SENS et 5-C.

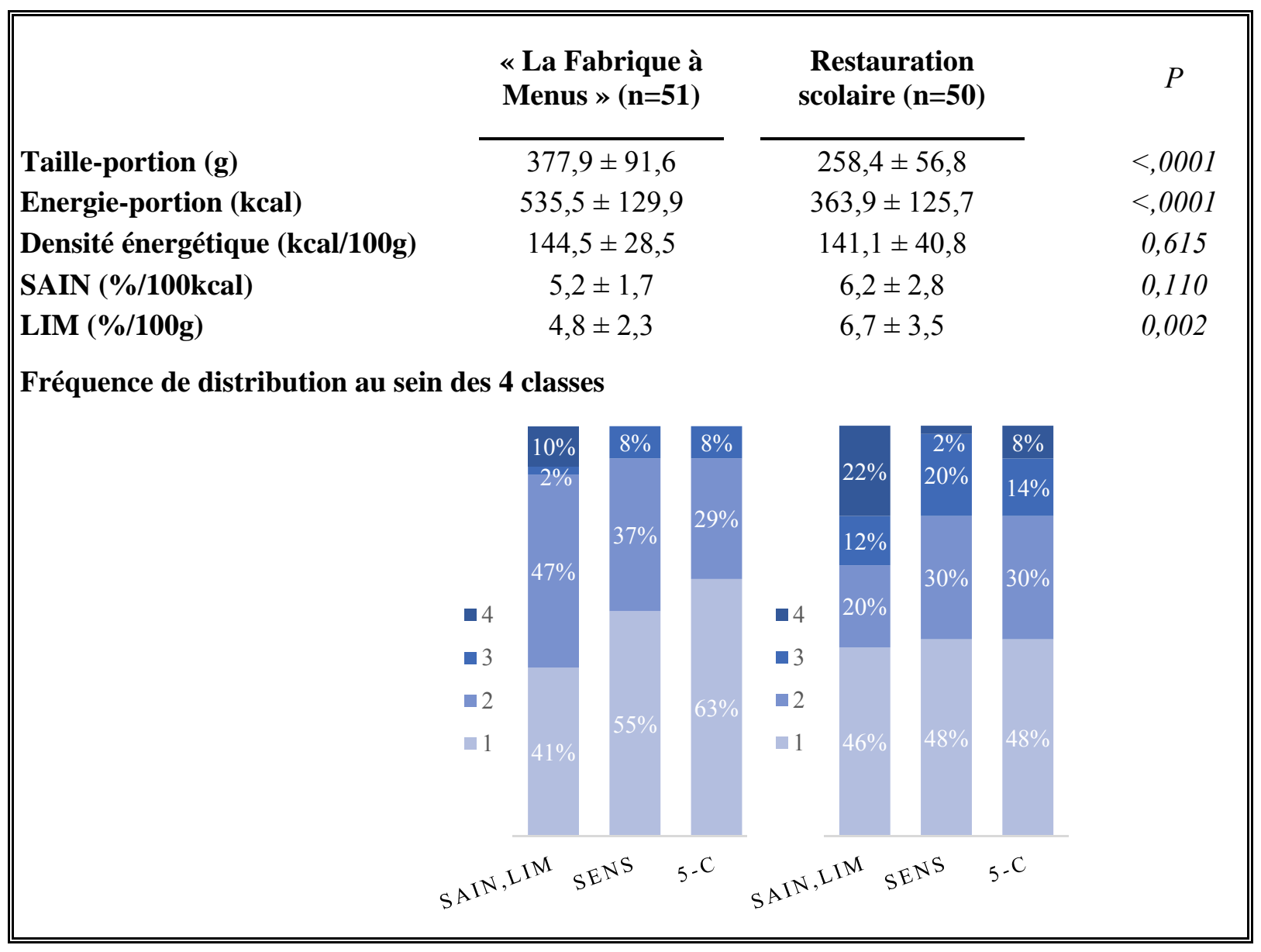

